PROCEEDINGS OF THE

AMERICAN MATHEMATICAL SOCIETY

Volume 137, Number 8, August 2009, Pages 2709-2718

S 0002-9939(09)09889-X

Article electronically published on March 10, 2009

\title{
HYPERCYCLICITY OF WEIGHTED CONVOLUTION OPERATORS ON HOMOGENEOUS SPACES
}

\author{
C. CHEN AND C-H. CHU
}

(Communicated by Nigel J. Kalton)

\begin{abstract}
Let $1 \leq p<\infty$. We show that a weighted translation operator on the $L^{p}$ space of a homogeneous space is hypercyclic under some condition on the weight. This condition is also necessary in the discrete case and is equivalent to hereditary hypercyclicity of the operator. The condition can be strengthened to characterise topologically mixing weighted translation operators on discrete spaces.
\end{abstract}

\section{INTRODUCTION}

Given a continuous linear self-map $T$ on a Fréchet space $X$, we denote its iterates by

$$
T^{1}=T, \ldots, \quad T^{n+1}=T^{n} \circ T, \quad \ldots \quad(n=1,2, \ldots) .
$$

The operator $T$ is said to be hypercyclic if there is a vector $x \in X$ such that the orbit $\left\{x, T x, \ldots, T^{n} x, \ldots\right\}$ is dense in $X$, in which case $x$ is called a hypercyclic vector for $T$. A Fréchet space admits a hypercyclic operator if, and only if, it is separable and infinite-dimensional [1, 6, 11. Hypercyclic operators have been studied by many authors since the work of Birkhoff 9 and MacLane 24]; we refer to 21, 22, for recent surveys and 2, 3, 7, 12, 14, 25, 26, for some recent works.

Let $\mathbb{N}$ denote the set of positive integers and $\mathbb{N}_{0}=\mathbb{N} \cup\{0\}$. Rolewicz [27] began the study of hypercyclic operators in classical Banach spaces and showed that a scalar multiple $\lambda B$ of the backward shift $B\left(x_{0}, x_{1}, \ldots\right)=\left(x_{1}, x_{2}, \ldots\right)$ on $\ell^{2}\left(\mathbb{N}_{0}\right)$ is hypercyclic for any complex number $\lambda$ with $|\lambda|>1$ while $B$ itself is not hypercyclic. Hypercyclicity of generalized backward shifts on Banach spaces has been considered in [20] and hypercyclic bilateral weighted shifts on $\ell^{2}(\mathbb{Z})$ have been characterised by Salas 28 in terms of their weights.

Following a recent study of convolution operators on groups and homogeneous spaces in 13, it is natural to consider the question of hypercyclicity for these operators. Hypercyclicity of convolution operators on spaces of ultradifferentiable functions has been studied in [10. Although Birkhoff's seminal result 9] shows the hypercyclicity of the translation operator on the space of entire functions, in contrast, a translation operator, or a convolution operator by a measure of unit mass, on $L^{p}$ spaces of locally compact groups is never hypercyclic. In this paper,

Received by the editors October 28, 2008.

2000 Mathematics Subject Classification. Primary 47A16, 47B37, 47B38, 43A85, 44 A35.

Key words and phrases. Hypercyclic operator, topologically mixing operator, convolution, homogeneous space, $L^{p}$-space. 
we give a sufficient condition for a weighted translation operator on the $L^{p}$ space of a homogeneous space to be hypercyclic. This condition is also necessary in the discrete case which subsumes the above result of Salas, and further, it is equivalent to hereditary hypercyclicity of the weighted translation operator. By strengthening the condition and analogous arguments, we also characterise topologically mixing weighted translation operators on discrete homogeneous spaces. Hypercyclcity in weighted $L^{p}$ spaces has also been studied in [16, 17.

We will make use of the following form of the hypercyclic criterion in [8], derived from the original one obtained by Kitai [23, and by Gethner and Shapiro [19] independently.

Lemma 1.1. Let $T$ be a continuous linear self-map on a Fréchet space $X$. Then $T$ is hypercyclic if it satisfies the following criteria:

(i) $\left(T^{n}\right)$ admits a subsequence $\left(T^{n_{k}}\right)$ converging to zero pointwise on a dense subset of $X$;

(ii) there is a dense subset $Y$ of $X$ and a sequence of maps $S_{n_{k}}: Y \rightarrow X$ such that $\left(S_{n_{k}}\right)$ tends to zero pointwise on $Y$ and $\left(T^{n_{k}} S_{n_{k}}\right)$ tends to the identity pointwise on $Y$.

We note that the above criteria are not necessary for hypercyclicity [4, 15, although they have been shown in [8] to be equivalent to $T \oplus T$ being hypercyclic and also equivalent to $T$ being hereditarily hypercyclic; that is, there is an increasing sequence $\left(n_{k}\right)$ in $\mathbb{N}$ such that every subsequence $\left(T^{m_{k}}\right)$ of $\left(T^{n_{k}}\right)$ admits a vector $z \in X$ for which the orbit $\left\{T^{m_{k}} z\right\}_{k=1}^{\infty}$ is dense in $X$. If, moreover, the sequence $\left(n_{k}\right)$ in the hypercyclic criterion satisfies the syndetic condition $\sup _{k}\left\{n_{k+1}-n_{k}\right\}<\infty$, then it has been shown in [14] that $T$ is topologically mixing; that is, given nonempty open sets $U, V \subset X$, there exists $N \in \mathbb{N}$ such that $T^{n}(U) \cap V \neq \emptyset$ for all $n>N$. In fact, an operator satisfies the hypercyclic criterion for a syndetic sequence if and only if it does so for the full sequence $(n)$ [5, Remark 3.3].

\section{Weighted CONVOLUTION OPERATORS}

In what follows, let $G$ be a locally compact second countable group with identity $e$ and a right-invariant Haar measure $\lambda$ which is the counting measure if $G$ is discrete. Then $G$ is a union of a nested sequence

$$
G_{1} \subset G_{2} \subset \cdots \subset G_{n} \subset \cdots
$$

of compact sets with $G_{n}$ contained in the interior of $G_{n+1}$. Let $G$ act transitively on a locally compact Hausdorff space $\Omega$ by a continuous right action

$$
(v, g) \in \Omega \times G \mapsto v g \in \Omega
$$

so that $\Omega$ is homeomorphic to, and hence identifies with, a right coset space $G / H$ of $G$ by an isotropy subgroup $H$. We assume throughout that $H$ is compact, in which case $G / H$ admits a $G$-invariant measure $\nu$ satisfying $\nu=\lambda \circ q^{-1}$ where $q: G \rightarrow G / H$ will always denote the quotient map [18, p.58]. We call $\Omega$ a homogeneous space of $G$ and consider convolution operators on $\Omega$.

Let $C_{c}(G / H)$ be the space of continuous functions on $G / H$ with compact support. Let $1 \leq p \leq \infty$ and let $L^{p}(\Omega)=L^{p}(G / H)$ be the complex Lebesgue space of $G / H$ with respect to $\nu$, and write $L^{p}(G)$ for $H=\{e\}$. Given a complex Borel 
measure $\sigma$ on $G$ with (finite) total variation $\|\sigma\|=|\sigma|(G)$, we define a convolution operator $T_{\sigma}: L^{p}(G / H) \longrightarrow L^{p}(G / H)$ by

$$
T_{\sigma}(f)=f * \sigma \quad\left(f \in L^{p}(G / H)\right),
$$

where the convolution

$$
f * \sigma(H x)=\int_{G} f\left(H x y^{-1}\right) d \sigma(y)
$$

exists $\nu$-almost everywhere and is defined to be 0 otherwise. The operator $T_{\sigma}$ is bounded with norm $\left\|T_{\sigma}\right\|_{p} \leq\|\sigma\|$. Hence $T_{\sigma}$ is not hypercyclic if $\|\sigma\| \leq 1$.

A continuous function $w: G \rightarrow(0, \infty)$ is called a weight for $G / H$ if it satisfies

$$
w(h x)=w(x) \quad(x \in G, h \in H)
$$

so that $w^{\prime}(H x):=w(x)$ is a well-defined function on $G / H$. If such a weight $w$ is in $L^{\infty}(G)$, we can define a weighted convolution operator

$$
T_{\sigma, w}: f \in L^{p}(G / H) \mapsto T_{\sigma, w} f \in L^{p}(G / H),
$$

where

$$
T_{\sigma, w} f(H x)=w(x)(f * \sigma)(H x) \quad\left(f \in L^{p}(G / H)\right) .
$$

The operator $T_{\sigma, w}$ is not hypercyclic if $\|\sigma\|\|w\|_{\infty} \leq 1$. One can also consider the weighted convolution operator $\widetilde{T}_{\sigma, w}: L^{p}(G) \longrightarrow L^{p}(G)$ with $\widetilde{T}_{\sigma, w} f=w(f * \sigma)$. It is a 'lift' of $T_{\sigma, w}$ in the following commutative diagram:

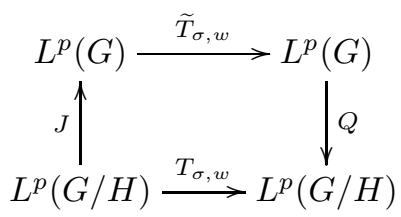

where the continuous linear maps $J: L^{p}(G / H) \longrightarrow L^{p}(G)$ and $Q: L^{p}(G) \longrightarrow$ $L^{p}(G / H)$ are injective and surjective respectively, with $\|Q\| \leq 1$, and are given by

$$
J f=f \circ q, \quad Q g(H x)=\int_{H} g(\xi x) d \xi \quad\left(f \in L^{p}(G / H), g \in L^{p}(G)\right)
$$

with $d \xi$ being the normalized Haar measure on the compact group $H$ (cf. [13]). We have $T_{\sigma, w} Q=Q \widetilde{T}_{\sigma, w}[13$, p. 77], which gives the following result.

Lemma 2.1. Let $1 \leq p<\infty$. If the weighted convolution operator $\widetilde{T}_{\sigma, w}$ possesses a hypercyclic vector $g \in L^{p}(G)$, then $Q g$ is a hypercyclic vector for $T_{\sigma, w}$ on $L^{p}(G / H)$.

For each $a \in G$, we denote by $\delta_{a}$ the unit mass at $a$ and by $\delta_{a}^{n}$ the $n$-fold convolution $\delta_{a} * \cdots * \delta_{a}$. We define $\delta_{a}^{0}=\delta_{e}$ and write $T_{a}$ for the convolution operator $T_{\delta_{a}}$.

Given a weight $w \in L^{\infty}(G)$ for $G / H$, the weighted convolution operator $T_{\delta_{a}, w}$ is written simply $T_{a, w}$, which is a weighted translation operator. If we also have $w^{-1} \in L^{\infty}(G)$, then the weighted convolution operator $T_{a^{-1}, w^{-1} * \delta_{a-1}}$ is the inverse of $T_{a, w}$. We write $S_{a, w}$ for $T_{a^{-1}, w^{-1} * \delta_{a-1}}$ to simplify notation. Thus, for each $f \in L^{p}(G / H)$, we have

$$
\begin{aligned}
& T_{a, w} f(H x)=w(x) f\left(H x a^{-1}\right), \\
& S_{a, w} f(H x)=\frac{1}{w(x a)} f(H x a) .
\end{aligned}
$$


Without the assumption of $w^{-1} \in L^{\infty}(G)$, we can still define the operator $S_{a, w}$ from the subspace $C_{c}(G / H)$ to $L^{p}(G / H)$, and we will use the same notation for this map since no confusion is likely. The same remark applies to $T_{a, w}$ if $w \notin L^{\infty}(G)$. Evidently, the translation operator $T_{a}$ is not hypercyclic. However if one considers the weighted convolution operator $T_{a, w}$, then hypercyclicity can occur for certain weights. Indeed, we are going to describe these weights for arbitrary homogeneous spaces $\Omega=G / H$ and show, for a discrete group $G$, that these are the only weights making $T_{a, w}$ hereditarily hypercyclic.

Let $p \in[1, \infty]$ with conjugate exponent $q$ and let $\langle\cdot, \cdot\rangle: L^{p}(\Omega) \times L^{q}(\Omega) \longrightarrow \mathbb{C}$ be the duality. A simple computation gives

$$
\left\langle T_{\sigma, w} f, g\right\rangle=\left\langle f, T_{\widetilde{\sigma}}(w g)\right\rangle \quad\left(f \in L^{p}(\Omega), g \in L^{q}(\Omega)\right)
$$

where $\widetilde{\sigma}$ is the measure $d \widetilde{\sigma}(x)=d \sigma\left(x^{-1}\right)$. Therefore the dual map $T_{\sigma, w}^{*}: L^{q}(\Omega) \longrightarrow$ $L^{q}(\Omega)$ is given by $T_{\sigma, w}^{*}(g)=T_{\widetilde{\sigma}}(w g)$ for $g \in L^{q}(\Omega)$. In particular, if $\sigma=\delta_{a}$, we have

$$
T_{a, w}^{*}(g)=T_{\delta_{a^{-1}}}(w g)=T_{a^{-1}, w * \delta_{a^{-1}}}(g) \quad\left(g \in L^{q}(\Omega)\right)
$$

and $T_{a, w}^{*}$ is a weighted convolution operator on $L^{q}(\Omega)$.

By a null set in $G$, we mean a Borel set $K \subset G$ with $\lambda(K)=0$.

Proposition 2.2. Let $G$ be a locally compact second countable group with $a \in G$. Let $\Omega=G / H$ be a homogeneous space of $G$. Let $w: G \rightarrow(0, \infty)$ be a weight for $G / H$ satisfying $w \in L^{\infty}(G)$. Let $1 \leq p<\infty$ and $T_{a, w}$ be the weighted convolution operator on $L^{p}(\Omega)$ defined above. Then condition (ii) below implies (i).

(i) $T_{a, w}$ is hereditarily hypercyclic.

(ii) Both sequences (depending on a)

$$
w_{n}:=\prod_{s=1}^{n} w * \delta_{a^{-1}}^{s} \quad \text { and } \quad \bar{w}_{n}:=\left(\prod_{s=0}^{n-1} w * \delta_{a}^{s}\right)^{-1}
$$

admit respectively subsequences $\left(w_{n_{k}}\right)$ and $\left(\bar{w}_{n_{k}}\right)$ which converge pointwise to $0 \quad \lambda$-a.e. and are uniformly bounded on each non-null compact subset $K$ of $G$.

Proof. Let $\left(w_{n_{k}}\right)$ and $\left(\bar{w}_{n_{k}}\right)$ be subsequences of $\left(w_{n}\right)$ and $\left(\bar{w}_{n}\right)$ respectively satisfying (ii). We show that $T_{a, w}$ satisfies the hypercyclic criterion.

We make use of the sequence of maps $S_{a, w}^{n_{k}}: C_{c}(G / H) \longrightarrow L^{p}(G / H)$. Let $f \in C_{c}(G / H) \backslash\{0\}$ with compact support supp $f$. Then we have $T_{a, w}^{n_{k}}\left(S_{a, w}^{n_{k}} f\right)=f$. We show that $\left\|T_{a, w}^{n_{k}} f\right\|_{p} \rightarrow 0$ as $n_{k} \rightarrow \infty$. There exists a compact set $K \subset G$ with $q(K)=\operatorname{supp} f$ (see, for instance, [18, 2.46]). It follows that $q^{-1}(\operatorname{supp} f)=$ $q^{-1}(q(K))=H K$, which is compact and non-null. Let $\left(w_{n_{k}}\right)$ be bounded on $H K$ by $M$ say. Let $\varepsilon>0$ and choose, by Egoroff's theorem, a Borel set $E \subset H K$ such that $\lambda(H K \backslash E)<\frac{\varepsilon}{M^{p}\|f\|_{\infty}^{p}}$ and $\left(w_{n_{k}}^{p}\right)$ converges to 0 uniformly on $E$. There exists 
$N \in \mathbb{N}$ such that $w_{n_{k}}^{p}<\frac{\varepsilon}{\|f\|_{p}^{p}}$ on $E$ for $n_{k}>N$. We have, by change of variables,

$$
\begin{aligned}
\| T_{a, w}^{n_{k} f \|_{p}^{p}} & =\int_{G / H}\left|T_{a, w}^{n_{k}} f(H x)\right|^{p} d \nu(H x) \\
& =\int_{(\operatorname{Supp} f) a^{n_{k}}}\left|w(x) w\left(x a^{-1}\right) \cdots w\left(x a^{-\left(n_{k}-1\right)}\right)\right|^{p}\left|f\left(H x a^{-n_{k}}\right)\right|^{p} d \nu(H x) \\
& =\int_{H K a^{n_{k}}}\left|w(x) w\left(x a^{-1}\right) \cdots w\left(x a^{-\left(n_{k}-1\right)}\right)\right|^{p}\left|f\left(H x a^{-n_{k}}\right)\right|^{p} d \lambda(x) \\
& =\int_{H K}\left|w\left(x a^{n_{k}}\right) w\left(x a^{n_{k}-1}\right) \cdots w(x a)\right|^{p}|f(H x)|^{p} d \lambda(x) \\
& =\int_{E} w_{n_{k}}^{p}(x)|f(H x)|^{p} d \lambda(x)+\int_{H K \backslash E} w_{n_{k}}^{p}(x)|f(H x)|^{p} d \lambda(x) \\
& \leq \frac{\varepsilon}{\|f\|_{p}^{p}}\|f\|_{p}^{p}+M^{p}\|f\|_{\infty}^{p} \lambda(H K \backslash E)<2 \varepsilon
\end{aligned}
$$

for $n_{k}>N$. Similar arguments using the sequence $\left(\bar{w}_{n_{k}}\right)$ yield

$$
\left\|S_{a, w}^{n_{k}} f\right\|_{p}^{p}=\int_{H K a^{-n_{k}}} \frac{1}{\left|w(x a) w\left(x a^{2}\right) \cdots w\left(x a^{n_{k}}\right)\right|^{p}}\left|f\left(H x a^{n_{k}}\right)\right|^{p} d \lambda(x) \longrightarrow 0 .
$$

Hence $T_{a, w}^{n_{k}}$ satisfies the hypercyclic criterion in Lemma 1.1 since $C_{c}(G / H)$ is dense in $L^{p}(G / H)$. Therefore $T_{a, w}$ is hereditarily hypercyclic by [8, Theorem 2.3].

Remark 2.3. In condition (ii) above, if the sequence $\left(n_{k}\right)$ is syndetic, then $T_{a, w}$ is topologically mixing by a remark after Lemma1.1 If we have $w^{-1} \in L^{\infty}(G)$ instead of $w \in L^{\infty}(G)$, then condition (ii) implies that $S_{a, w}$ is hereditarily hypercyclic on $L^{p}(G / H)$ by changing the roles of $T_{a, w}$ and $S_{a, w}$ in the above proof.

We note that, if $a=e$, then condition (ii) in Proposition 2.2 fails and, in fact, we have $\bar{w}_{n}=w_{n}^{-1}$ in this case. Also, a pointwise convergence sequence of continuous functions need not be uniformly bounded on a compact set. For example, the sequence $w_{n}(x)=2 n^{2} x e^{-n^{2} x^{2}}$ is not uniformly bounded on $[0,1]$.

There are examples of hypercyclic operators with hypercyclic dual [26, 28]. The following result shows that $T_{a, w}$ and its dual $T_{a, w}^{*}$ can both be hypercyclic for certain weights $w$.

Corollary 2.4. The dual $T_{a, w}^{*}$ of a weighted translation operator $T_{a, w}: L^{p}(G / H)$ $\longrightarrow L^{p}(G / H)$ is hypercyclic if the weight $w * \delta_{a^{-1}}$ satisfies condition (ii) for $a^{-1}$ in Proposition 2.2 .

Example 2.5. Fix $t \in(0,1)$. We define a weight $w: \mathbb{R} \rightarrow(0, \infty)$ for $\mathbb{R}$ by

$$
w(x)= \begin{cases}t & \text { if } 1 \leq x \\ t^{x} & \text { if }-1 \leq x \leq 1 \\ \frac{1}{t} & \text { if } x \leq-1\end{cases}
$$

Then $w$ and $w^{-1}$ are bounded and continuous on $\mathbb{R}$, with $w$ satisfying condition (ii) for $a>0$ in Proposition 2.2. Indeed, let $K=[b, c]$ say. Pick $n_{0} \in \mathbb{N}$ such that $b+n_{0} a>1$. Since $w$ is decreasing, we have

$$
\begin{aligned}
0 & <w_{n}(x)=w(x+a) w(x+2 a) \cdots w(x+n a) \\
& \leq w(b+a) w(b+2 a) \cdots w(b+n a) \\
& \leq w(b+a) w(b+2 a) \cdots w\left(b+n_{0} a\right) \quad\left(x \in K, n \geq n_{0}\right) .
\end{aligned}
$$


It follows that $\left(w_{n}\right)$ is uniformly bounded on $K$ by some constant $M$. For each $x \in[b, c]$, we have $w(x+s)=t$ for all $s \geq n_{0} a$. This implies, for $n>n_{0}$,

$$
\begin{aligned}
w_{n}(x) & =w(x+a) w(x+2 a) \cdots w\left(x+n_{0} a\right) w\left(x+\left(n_{0}+1\right) a\right) \cdots w(x+n a) \\
& \leq M t^{n-n_{0}} \rightarrow 0 \text { as } n \rightarrow \infty .
\end{aligned}
$$

Hence $\left(w_{n}\right)$ converges to 0 uniformly on $[b, c]$. For the sequence $\left(\bar{w}_{n}\right)$, we have

$$
\begin{aligned}
\bar{w}_{n}(x) & =\frac{1}{w(x) w(x-a) \cdots w(x-(n-1) a)} \\
& \leq \frac{1}{w(c) w(c-a) \cdots w(c-(n-1) a)} \\
& \leq \frac{1}{w(c) w(c-a) \cdots w\left(c-\left(n_{1}-1\right) a\right)} \quad\left(x \in K, n \geq n_{1}\right),
\end{aligned}
$$

where $n_{1}$ is chosen so that $c-\left(n_{1}-1\right) a<-1$. Likewise, $\left(\bar{w}_{n}\right) \rightarrow 0$ uniformly on $[b, c]$.

In fact, the above example is a special case of the following lemma.

Lemma 2.6. Let $w$ be a weight for a locally compact second countable group $G$. The following conditions are equivalent.

(i) Given $\varepsilon>0$, a compact set $D \subset G$ and $N \in \mathbb{N}$, there exists $m>N$ satisfying $w_{m}(x)<\varepsilon$ and $\bar{w}_{m}(x)<\varepsilon$ for all $x \in D$.

(ii) Both sequences $\left(w_{n}\right)$ and $\left(\bar{w}_{n}\right)$ admit subsequences $\left(w_{n_{k}}\right)$ and $\left(\bar{w}_{n_{k}}\right)$ which converge uniformly to 0 on each compact subset $K$ of $G$.

If $G$ is discrete, then $D$ can be replaced by a singleton.

Proof. We show (i) $\Rightarrow$ (ii). Since $G$ is a union $\bigcup_{k=1}^{\infty} G_{k}$ of nested compact sets $G_{k}$ with $G_{k}$ contained in the interior of $G_{k+1}$, it suffices to prove convergence on $G_{t}$ for each $t \in \mathbb{N}$.

Let $\varepsilon=\frac{1}{2}$ and $D=G_{1}$. Then there exists $n_{1}$ such that $w_{n_{1}}(x)<\frac{1}{2}$ and $\bar{w}_{n_{1}}(x)<\frac{1}{2}$ for all $x \in G_{1}$. Inductively, for each $k>1$, there exists $n_{k}>n_{k-1}$ such that $w_{n_{k}}(x)<\frac{1}{2^{k}}$ and $\bar{w}_{n_{k}}(x)<\frac{1}{2^{k}}$ for all $x \in G_{k}$.

Now let $\varepsilon>0$ and choose $k_{0} \in \mathbb{N}$ with $k_{0}>t$ and $\frac{1}{2^{k_{0}}}<\varepsilon$. Then, for all $k>k_{0}$, we have

$$
w_{n_{k}}(x)<\frac{1}{2^{k}}<\frac{1}{2^{k_{0}}}<\varepsilon \text { and } \bar{w}_{n_{k}}(x)<\varepsilon
$$

on $G_{k} \supset G_{t}$. Hence $\left(w_{n_{k}}\right)$ and $\left(\bar{w}_{n_{k}}\right)$ converge uniformly to 0 on $G_{t}$.

We now consider discrete groups and derive necessary and sufficient conditions for a weighted translation operator to be hypercyclic. A torsion element of a group $G$ is an element of finite order.

Lemma 2.7. Let $G$ be a discrete group and $a \in G$. Then $a$ is not a torsion element if and only if for any finite subset $D \subset G$, there exists $N \in \mathbb{N}$ such that $D \cap D a^{ \pm n}=\emptyset$ for $n>N$.

Proof. Given that $a$ is not a torsion element, we observe that, for every $d \in D$, there exists $N_{d}$ such that $d a^{n} \notin D$ for $n>N_{d}$. Otherwise, there is some $d \in D$ such that $d a^{n_{j}} \in D$ for a strictly increasing sequence $\left(n_{j}\right)$ in $\mathbb{N}$. Since $D$ is finite, we must have $d a^{n_{j}}=d a^{n_{t}}$ for some $n_{j} \neq n_{t}$, which contradicts the fact that $a$ is not a torsion element. Let $N=\max \left\{N_{d}: d \in D\right\}$. Then $D \cap D a^{n}=\emptyset$ for $n>N$. The condition $D \cap D a^{-n}=\emptyset$ can be proved similarly. 
On the other hand, if $a \in G$ is a torsion element with order $m$, then for any finite subset $D \subset G$, there exist infinitely many $n$ 's such that $D \cap D a^{n} \neq \emptyset$. Indeed, $D \cap D a^{n}=D \neq \emptyset$ for $n \in m \mathbb{Z}$.

Theorem 2.8. Let $G$ be a discrete group and $H$ a finite subgroup. Choose $a \in G$ that is not a torsion element. Let $w: G \rightarrow(0, \infty)$ be a weight for $G / H$ such that $w \in \ell^{\infty}(G)$. Let $1 \leq p<\infty$ and $T_{a, w}$ be the weighted convolution operator on $\ell^{p}(G / H)$ defined by $a$ and $w$. The following conditions are equivalent.

(i) $T_{a, w}$ is hypercyclic.

(ii) $T_{a, w}$ is hereditarily hypercyclic.

(iii) Both sequences (depending on a)

$$
w_{n}=\prod_{s=1}^{n} w * \delta_{a^{-1}}^{s} \quad \text { and } \quad \bar{w}_{n}=\left(\prod_{s=0}^{n-1} w * \delta_{a}^{s}\right)^{-1}
$$

admit subsequences $\left(w_{n_{k}}\right)$ and $\left(\bar{w}_{n_{k}}\right)$ which converge to 0 pointwise in $G$.

In particular, if $G$ is torsion free, then the above conditions are equivalent for all $a \in G \backslash\{e\}$.

Proof. By Proposition [2.2, (iii) implies (i) and (ii) since a compact subset of a discrete group is finite. We only need to show (i) implies (iii).

Let $T_{a, w}$ be hypercyclic. Let $\varepsilon>0$ and $z \in G$. Fix $N \in \mathbb{N}$. Let $\chi_{z} \in \ell^{p}(G / H)$ be the characteristic function

$$
\chi_{z}(H x)= \begin{cases}1 & \text { if } x \in H z \\ 0 & \text { otherwise. }\end{cases}
$$

Choose $0<\delta<\frac{\varepsilon}{1+\varepsilon}$. Since the set of hypercyclic vectors for $T_{a, w}$ is dense, there exist a hypercyclic vector $f \in \ell^{p}(G / H)$ for $T_{a, w}$ and some $m>N$ such that

$$
\left\|f-\chi_{z}\right\|_{p}<\delta \text { and }\left\|T_{a, w}^{m} f-\chi_{z}\right\|_{p}<\delta .
$$

By Lemma 2.7, we may choose $m$ sufficiently large so that $H z \cap H z a^{ \pm m}=\emptyset$. Since

$$
\left\|f-\chi_{z}\right\|_{p}^{p}=\sum_{H x \in G / H}\left|f(H x)-\chi_{z}(H x)\right|^{p} \nu(H x)=\sum_{x \in G}\left|f(H x)-\chi_{z}(H x)\right|^{p}<\delta^{p},
$$

where $\nu(H x)=\lambda(H)$, we have

$$
\left|f(H x)-\chi_{z}(H x)\right|<\delta \quad(x \in G) .
$$

This gives

$$
\begin{array}{ll}
|f(H x)|>1-\delta & \text { for } x \in H z \\
|f(H x)|<\delta & \text { for } x \notin H z .
\end{array}
$$

From $\left\|T_{a, w}^{m} f-\chi_{z}\right\|_{p}<\delta$, we also deduce that

$$
\left|w(x) w\left(x a^{-1}\right) \cdots w\left(x a^{-(m-1)}\right) f\left(H x a^{-m}\right)-\chi_{z}(H x)\right|<\delta \quad(x \in G) .
$$

In particular,

$$
\bar{w}_{m}(z)^{-1}\left|f\left(H z a^{-m}\right)\right|>1-\delta .
$$

Since $H z \cap H z a^{-m}=\emptyset$, we have

$$
\bar{w}_{m}(z)<\frac{\left|f\left(H z a^{-m}\right)\right|}{1-\delta}<\frac{\delta}{1-\delta}<\varepsilon .
$$


From (2), we have

$$
\left|w\left(x a^{m}\right) w\left(x a^{m-1}\right) \cdots w(x a) f(H x)-\chi_{z}\left(H x a^{m}\right)\right|<\delta \quad(x \in G)
$$

and hence, as $H z \cap H z a^{m}=\emptyset$, one obtains

$$
w_{m}(z)|f(H z)|<\delta .
$$

It follows that

$$
w_{m}(z)<\frac{\delta}{|f(H z)|}<\frac{\delta}{1-\delta}<\varepsilon .
$$

This proves that $\left(w_{n}\right)$ and $\left(\bar{w}_{n}\right)$ satisfy condition (i) in Lemma 2.6 for each point $z \in$ $G$, and hence they admit subsequences $\left(w_{n_{k}}\right)$ and $\left(\bar{w}_{n_{k}}\right)$ which converge pointwise to 0 on $G$.

Remark 2.9. The above result implies that if $T_{a, w}: \ell^{p}(G / H) \longrightarrow \ell^{p}(G / H)$ is hypercyclic for some $p \in[1, \infty)$, then it is so for all $p \in[1, \infty)$. As in Remark 2.3, if $w^{-1} \in \ell^{\infty}(G)$, then Theorem 2.8 applies to $S_{a, w}$. We also note that torsion freeness is not required in Theorem 2.8 for the case of $\ell^{p}(G)$ since $H z$ reduces to $\{z\}$ in the above proof.

Corollary 2.10. Let $a \in G$ and $w \in \ell^{\infty}(G)$ be as in Theorem 2.8 for the homogeneous space $G / H$. Then $T_{a, w}: \ell^{p}(G / H) \longrightarrow \ell^{p}(G / H)$ is hypercyclic if and only if the lift $\widetilde{T}_{a, w}: \ell^{p}(G) \longrightarrow \ell^{p}(G)$ is hypercyclic.

Proof. This follows from Lemma 2.1 and Theorem 2.8

Example 2.11. The weighted shift with weight sequence $\left(a_{n}\right)$ studied in 28 is the weighted convolution operator $S_{a, w}$ on $\ell^{2}(\mathbb{Z})$ with $a=-1 \in \mathbb{Z}$ and the weight $w(n)=a_{n}^{-1}$. By Remark [2.9] and Lemma 2.6] $S_{a, w}$ is hypercyclic if and only if given $\varepsilon>0$ and $q \in \mathbb{N}$, there exists an arbitrarily large $n$ such that for all $|j| \leq q$, we have

$$
\prod_{s=1}^{n} w(j-s)=w_{n}(j)<\varepsilon \quad \text { and } \quad \prod_{s=0}^{n-1} w(j+s)=\bar{w}_{n}(j)^{-1}>\frac{1}{\varepsilon},
$$

which is the condition in [28, Theorem 2.1].

Using similar arguments as in the proof of Theorem 2.8, one can also characterise topologically mixing weighted translation operators on $\ell^{p}(G / H)$, which extends a result in [14, Theorem 1.2] for $\ell^{2}(\mathbb{Z})$.

Corollary 2.12. Let $T_{a, w}$ be the operator defined in Theorem 2.8, The following conditions are equivalent.

(i) $T_{a, w}$ is topologically mixing.

(ii) Both sequences (depending on a)

$$
w_{n}=\prod_{s=1}^{n} w * \delta_{a^{-1}}^{s} \quad \text { and } \quad \bar{w}_{n}=\left(\prod_{s=0}^{n-1} w * \delta_{a}^{s}\right)^{-1}
$$

converge to 0 pointwise in $G$.

If $G$ is torsion free, then the above conditions are equivalent for all $a \in G \backslash\{e\}$. 
Proof. We see from Remark 2.3 that condition (ii) implies (i). For the converse, let $\varepsilon>0$ and fix $z \in G$ with the characteristic function $\chi_{z} \in \ell^{p}(G / H)$ as defined in the proof of Theorem 2.8. Choose $0<\delta<\frac{\varepsilon}{1+\varepsilon}$ and let $U=\left\{f \in \ell^{p}(G / H)\right.$ : $\left.\left\|f-\chi_{z}\right\|<\delta\right\}$. By the topologically mixing assumption, there exists $N \in \mathbb{N}$ such that

$$
T_{a, w}^{n}(U) \cap U \neq \emptyset \quad(n>N) .
$$

We can therefore pick, for each $n>N$, a function $f_{n} \in U$ with $T_{a, w}^{n} f_{n} \in U$ which gives

$$
\left\|f_{n}-\chi_{z}\right\|_{p}<\delta \text { and }\left\|T_{a, w}^{n} f_{n}-\chi_{z}\right\|_{p}<\delta .
$$

Using this for each $f_{n}$ and repeating the arguments in the proof of Theorem 2.8, we arrive at

$$
\bar{w}_{n}(z)<\varepsilon \text { and } w_{n}(z)<\varepsilon
$$

for all $n>N$, proving that $\left(w_{n}\right)$ and $\left(\bar{w}_{n}\right)$ converge to 0 pointwise in $G$.

We conclude with a remark that the weighted translation operator $T_{a, w}$ above and its dual $T_{a, w}^{*}$ can never be simultaneously topologically mixing since $T_{a, w}^{*}=$ $T_{a^{-1}, w * \delta_{a^{-1}}}$, and for $a^{-1} \in G$ the two sequences for the weight $w * \delta_{a^{-1}}$ in condition (ii) above are given by

$$
\left(w * \delta_{a^{-1}}\right)_{n}=\prod_{s=1}^{n}\left(w * \delta_{a^{-1}}\right) * \delta_{a}^{s}=\bar{w}_{n}^{-1}
$$

and $\left(\overline{w * \delta_{a^{-1}}}\right)_{n}=w_{n}^{-1}$.

\section{REFERENCES}

[1] S.I. Ansari, Existence of hypercyclic operators on topological vector spaces, J. Funct. Anal. 148 (1997) 384-390. MR1469346 (98h:47028a)

[2] F. Bayart and S. Grivaux, Frequently hypercyclic operators, Trans. Amer. Math. Soc. 358 (2006) 5083-5117. MR2231886 (2007e:47013)

[3] F. Bayart and S. Grivaux, Invariant Gaussian measures for operators on Banach spaces and linear dynamics, Proc. Lond. Math. Soc. (3) 94 (2007) 181-210. MR2294994 (2008i:47019)

[4] F. Bayart and É. Matheron, Hypercyclic operators failing the hypercyclicity criterion on classical Banach spaces, J. Funct. Anal. 250 (2007) 426-441. MR2352487(2008k:47016)

[5] T. Bermúdez, A. Bonilla, J.A. Conejero and A. Peris, Hypercyclic, topologically mixing and chaotic semigroups on Banach spaces, Studia Math. 170 (2005) 57-75. MR2142183 (2005m:47013)

[6] L. Bernal-González, On hypercyclic operators on Banach spaces, Proc. Amer. Math. Soc. 127 (1999) 1003-1010. MR.1476119 (99f:47010)

[7] L. Bernal-González and K.-G. Grosse-Erdmann, The hypercyclicity criterion for sequences of operators, Studia Math. 157 (2003) 17-32. MR.1980114 (2003m:47013)

[8] J. Bès and A. Peris, Hereditarily hypercyclic operators, J. Funct. Anal. 167 (1999) 94-112. MR.1710637 (2000f:47012)

[9] G.D. Birkhoff, Démonstration d'un théorème élémentaire sur les fonctions entières, C.R. Acad. Sci. Paris 189 (1929) 473-475.

[10] J. Bonet, Hypercyclic and chaotic convolution operators, J. London Math. Soc. (2) 62 (2000) 253-262. MR:1772185 (2001g:47053)

[11] J. Bonet and A. Peris, Hypercyclic operators on non-normable Fréchet spaces, J. Funct. Anal. 159 (1998) 587-595. MR1658096 (99k:47044)

[12] J-C. Chen and S-Y. Shaw, Topological mixing and hypercyclicity criterion for sequences of operators, Proc. Amer. Math. Soc. 134 (2006) 3171-3179. MR2231900 (2007d:47008)

[13] C-H. Chu, Matrix convolution operators on groups, Lecture Notes in Math. 1956, SpringerVerlag, Berlin-Heidelberg, 2008. MR2450997 
[14] G. Costakis and M. Sambarino, Topologically mixing hypercyclic operators, Proc. Amer. Math. Soc. 132 (2004) 385-389. MR2022360 (2004i:47017)

[15] M. De La Rosa and C. Read, A hypercyclic operator whose direct sum $T \oplus T$ is not hypercyclic, J. Operator Theory (to appear).

[16] R. deLaubenfels and H. Emamirad, Chaos for functions of discrete and continuous weighted shift operators, Ergod. Th. Dynam. Sys. 21 (2001) 1411-1427. MR.1855839 (2002j:47030)

[17] W. Desch, W. Schappacher and G. Webb, Hypercyclic and chaotic semigroups of linear operators, Ergod. Th. Dynam. Sys. 17 (1997) 793-819. MR1468101 (98j:47083)

[18] G.B. Folland, A course in abstract harmonic analysis, CRC Press, Boca Raton, FL, 1995. MR.1397028 (98c:43001)

[19] R.M. Gethner and J.H. Shapiro, Universal vectors for operators on spaces of holomorphic functions, Proc. Amer. Math. Soc. 100 (1987) 281-288. MR884467(88g:47060)

[20] G. Godefroy and J.H. Shapiro, Operators with dense, invariant, cyclic vector manifolds, J. Funct. Anal. 98 (1991) 229-269. MR1111569 (92d:47029)

[21] K.-G. Grosse-Erdmann, Universal families and hypercyclic operators, Bull. Amer. Math. Soc. (N.S.) 36 (1999) 345-381. MR.1685272 (2000c:47001)

[22] K.-G. Grosse-Erdmann, Recent developments in hypercyclicity, RACSAM Rev. R. Acad. Cienc. Exactas Fis. Nat. Ser. A Mat. 97 (2003) 273-286. MR2068180 (2005c:47010)

[23] C. Kitai, Invariant closed sets for linear operators, PhD Thesis, University of Toronto, 1982.

[24] G.R. MacLane, Sequences of derivatives and normal families, J. Analyse Math. 2 (1952) 72-87. MR0053231(14:741d)

[25] A. Peris and L. Saldivia, Syndetically hypercyclic operators, Integr. Equ. Oper. Theory 51 (2005) 275-281. MR2120081 (2005h:47017)

[26] H. Petersson, Spaces that admit hypercyclic operators with hypercyclic adjoints, Proc. Amer. Math. Soc. 134 (2006) 1671-1676. MR2204278 (2007b:47020)

[27] S. Rolewicz, On orbits of elements, Studia Math. 32 (1969) 17-22. MR0241956 (39:3292)

[28] H. Salas, Hypercyclic weighted shifts, Trans. Amer. Math. Soc. 347 (1995) 993-1004. MR.1249890 (95e:47042)

School of Mathematical Sciences, Queen Mary, University of London, London E1 4NS, United KINGDOM

E-mail address: c.chen@qmul.ac.uk

School of Mathematical Sciences, Queen Mary, University of London, London E1 4NS, United Kingdom

E-mail address: c.chu@qmul.ac.uk 